\title{
MEJORAMIENTO DEL RENDIMIENTO Y CALIDAD DE FRUTO Y PSEUDOFRUTO DE MARAÑON CON UN CICLO DE FERTILIZACIÓN ORGÁNICA ${ }^{1}$
}

\author{
LOURDES ADRIANO-ANAYA², GUADALUPE TREJO-ROBLERO 3 , \\ RAYMUNDO ROSAS-QUIJANO ${ }^{4}$, GAMALIEL VELÁZQUEZ-OVALLE 5 , \\ ALFREDO VÁZQUEZ-OVANDO 6
}

RESUMEN-El objetivo del presente estudio fue evaluar la influencia de la aplicación de fertilizantes orgánicos a árboles adultos de marañón por un ciclo productivo sobre la producción y calidad de frutos y pseudofrutos. Para lo anterior, se establecieron tres tratamientos: fertilización orgánica (BIO), fertilización sintética (FS) y sin fertilización (SF). Se cuantificó el número de panículas $\mathrm{m}^{-3}$, número de flores por panícula y el número de frutos $\mathrm{m}^{-3}$ en los árboles. Después del corte, a frutos y pseudofrutos se analizaron propiedades físicoquímicas de interés. Aunque el tratamiento FS promovió mayor número de panículas por unidad de área foliar $(\mathrm{P}<0.05)$ sobre los otros tratamientos, el rendimiento fue igual $(\mathrm{P}>0.05)$, medido como la cantidad de fruto-pseudofruto $\mathrm{m}^{-3}$ con el tratamiento BIO. Sin embargo, la cantidad de fruto (principal producto comercializado) fue mayor en $\mathrm{BIO}\left(1.80 \mathrm{~kg}^{2}\right.$ arbol $\left.^{-1}\right)$ comparado con los otros dos tratamientos (FS $=1.35 ; \mathrm{SF}$ $=1.09$ ). El tratamiento BIO aumentó en $81 \%$ el contenido de polifenoles respecto al tratamiento no fertilizado. Igualmente, los contenidos de grasa y acidez titulable fueron superiores en $\mathrm{BIO}$, respecto a los tratamientos sin fertilización y con fertilización sintética $(\mathrm{P}<0.05)$. Se demostró que la aplicación de biofertilizantes por un ciclo de producción aumenta la producción de frutos y modifica la composición química de las semillas. Términos para indexación: SST, lípidos, actividad antioxidante, Anacardium occidentale, biofertilizantes.

\section{A CYCLE OF ORGANIC FERTILIZATION OF CASHEW TREES IMPROVES THE YIELD AND QUALITY OF THE PRODUCTS}

\begin{abstract}
This study aimed to evaluate the effect of organic fertilizers on production and quality of fruits and pseudo fruits on adult trees of Anacardium occidentale L. by a productive cycle. We established three treatments; organic fertilization (BIO), synthetic fertilization (SF), and unfertilized (UF). The number of panicles $\mathrm{m}^{-3}$, number of flowers per panicle and the number of fruits $\mathrm{m}^{-3}$ in the trees was measured. After harvest, physicochemical analyses were performed to fruits and pseudo fruits. Although the SF treatment promoted a greater number of panicles per unit leaf area $(\mathrm{P}<0.05)$ on the other treatments, the yield (measured both as the amount of fruit-pseudo fruit $\left.\mathrm{m}^{-3}\right)$ was the same $(\mathrm{P}>0.05)$ with $\mathrm{BIO}$ treatment. However, the amount of fruit (main marketed product) was higher in BIO $\left(1.80 \mathrm{~kg} \mathrm{tree}^{-1}\right)$ compared to the other two treatments $(\mathrm{SF}=1.35 ; \mathrm{UF}=1.09)$. The BIO treatment increased by $81 \%$ polyphenol content compared to unfertilized treatment. Similarly, fat content and titratable acidity were higher in BIO, compared to treatments without fertilization and synthetic fertilization $(\mathrm{P}<0.05)$. It was demonstrated that the application of biofertilizer in a production cycle increases fruit production and modifies the chemical composition of seeds.
\end{abstract}

Index terms: TSS, lipids, antioxidant activity, Anacardium occidentale L., biofertilizers

\footnotetext{
1(Paper 085-16). Received June 28, 2016. Accepted October 07, 2016.

${ }^{2}$ Dra. en Agricultura Tropical, Instituto de Biociencias, Universidad Autónoma de Chiapas, México. E-mail: maria.adriano@unach.mx ${ }^{3}$ Ingeniero Biotecnólogo, Instituto de Biociencias, Universidad Autónoma de Chiapas. México. E-mail: fari_27092@hotmail.com ${ }^{4}$ Dr. en Biotecnología de Plantas, Instituto de Biociencias, Universidad Autónoma de Chiapas. México. E-mail: rrquijano@yahoo.fr ${ }^{5}$ Maestro en Biotecnología. Instituto de Biociencias, Universidad Autónoma de Chiapas. México. E-mail: gamavlz@gmail.com ${ }^{6}$ Dr. en Ciencias Biológicas. Instituto de Biociencias, Universidad Autónoma de Chiapas. Boulevard Príncipe Akishino sin número Colonia Solidaridad 2000, CP 30798. Tapachula, Chiapas, México. E-mail: jose.vazquez@unach.mx
} 


\section{INTRODUCCIÓN}

El marañón (Anacardium occidentale L.) es originario del noreste de Brasil, pertenece a la familia de las Anacardiáceas y actualmente está distribuido por Centro y Sudamérica (CATARINO et al., 2015). La semilla, el principal producto comercial obtenido de este cultivo, está contenido en un aquenio reniforme, el cual se encuentra adherido al pseudofruto que es conocido como la "manzana" del marañón (CATARINO et al., 2015). En México, el cultivo de marañón tiene importancia comercial principalmente de tipo local. Al sur del país, en el estado de Chiapas, esta anacardiácea se produce principalmente en la región Soconusco. En 2014, se produjeron 4226.53 ton de marañón (SIAP, 2016) en una superficie de 1436.5 ha. Esto da un rendimiento cercano a 3 ton ha ${ }^{-1}$, el cual resulta aceptable, pero inferior a los valores sugeridos para plantaciones de alta productividad reportadas en Brasil (XAVIER et al., 2013).

Pese a la gran demanda mundial de este producto, la producción en México no ha aumentado significativamente, por el contrario, en los últimos años ha disminuido. Una de las causas más influyentes para este fenómeno es la falta de industrialización y comercialización del pseudofruto, que en otras latitudes encuentra utilidad como materia prima para la obtención de jugos, antioxidantes, pigmentos y otros (DENDENA; CORSI, 2014). Aunado a esto, las plantaciones se ven afectadas por prácticas agrícolas deficientes, lo que limita el rendimiento sostenido. Además, por la fisiología de las plantas, las parcelas están establecidas en las proximidades de la línea costera, donde la salinidad es alta y el contenido de materia orgánica del suelo se encuentra disminuido. Por lo anterior, para elevar la producción se requiere de esquemas eficientes de fertilización.

El uso de fertilizantes sintéticos resulta la primera opción, sin embargo su aplicación se encuentra cada vez más comprometida, principalmente por el impacto negativo en las características del suelo, al promover mayor salinidad (DEINLEIN et al., 2014), pérdida de la microbiota (GEISSELER; SCOW, 2014), acomplejamiento de minerales disponibles para las plantas, eutrofización de cuerpos de agua y otras. Por el contrario, el uso de fertilizantes orgánicos como compost, lombricompost, lixiviados, bioles y materia verde, son aplicados en semillas, suelos o áreas de composteo con el objetivo de acelerar los procesos microbianos e incrementar la disponibilidad de nutrientes en forma tal que sean fácilmente asimilados por las plantas (THIRKELL et al., 2016). Además, han demostrado tener menor impacto ambiental y mayores beneficios tanto sociales como para la nutrición vegetal. En diversos cultivos se ha demostrado que su empleo puede garantizar la calidad de los frutos y formar parte de una tecnología que ofrece productividad biológica, económica y ecológica (JAVANMARDI; GHORBANI, 2012).

Diversos trabajos han referido que el empleo de biofertilizantes e inóculos microbianos, mejoran el aspecto de plantas de interés alimenticio, así como de los productos que de estas se obtienen (HERNÁNDEZ-FERNÁNDEZ et al., 2016; RIBEIRO et al., 2012). En plantas anuales, el efecto de los fertilizantes orgánicos puede apreciarse de manera rápida, pocos meses después de la siembra, sin embargo con plantas perennes el efecto de los fertilizantes puede tardar mucho tiempo en reflejarse (SONG et al., 2012) y no existen reportes que muestran el efecto inmediato de la aplicación de bio-productos sobre árboles adultos; por lo anterior, el objetivo del estudio fue evaluar el efecto de la fertilización orgánica por un ciclo en la producción y calidad, de fruto y pseudofruto de plantas adultas de marañón.

\section{MATERIALES Y MÉTODOS}

El estudio se llevó a cabo en una parcela con un área confinada de $13000 \mathrm{~m}^{2}(130 \mathrm{~m} \times 100 \mathrm{~m})$, localizada en el municipio de Villa Mazatán, Chiapas México $\left(14^{\circ} 45^{\prime} 52^{\prime \prime} \mathrm{N}, 92^{\circ} 28^{\prime} 14^{\prime \prime} \mathrm{O}\right.$, altitud de 7 msnm) durante el ciclo productivo comprendido del mes septiembre de 2012 a octubre de 2013. La edad promedio de los árboles fue de 20 años y sin fertilización de ningún tipo desde hace diez años.

Bajo un diseño de bloques completos al azar, se establecieron tres bloques de $4330 \mathrm{~m}^{2}$ cada uno. Con distribución aleatoria, se arreglaron tres tratamientos replicados en los tres bloques, los cuales corresponden: a) Fertilización orgánica (BIO), empleándose lixiviado de lombriz (humus) y biofermento líquido (Biol); b) Fertilización sintética (FS), empleando insumos sintéticos triple 18 y fosfato de amonio y c) sin fertilización (SF). Cada tratamiento estuvo conformado de 36 árboles (distribuidos en los tres bloques). La distancia entre árboles fue de $3 \mathrm{~m}$. Entre tratamientos del mismo bloque y entre bloques, se dejó un margen $6 \mathrm{~m}$ correspondiente a dos surcos de árboles.

El biofermento líquido empleado fue elaborado siguiendo el procedimiento descrito por Adriano et al. (2012). La composición promedio del biol $\left(\mathrm{p} \mathrm{v}^{-1}\right)$ fue: $1.5 \%$ de nitrógeno total, $0.98 \%$ 
de $\mathrm{P}_{2} \mathrm{O}_{5}$ y $1.04 \%$ de $\mathrm{K}_{2} \mathrm{O}$. Este producto se aplicó a razón de $40 \mathrm{~L}$ por árbol, en la zona de goteo, cada 14 días. Previo a la aplicación, el biol fue diluido con agua corriente hasta lograr una conductividad eléctrica promedio de $2.0 \mathrm{dS} \mathrm{m}^{-1}$.

El lixiviado del proceso de lombricompostaje (humus) fue obtenido según lo sugerido por Hernández-Fernández et al., (2016). La composición promedio del lixiviado $\left(\mathrm{p} \mathrm{v}^{-1}\right)$ fue $2.20 \%$ de nitrógeno total, $2.33 \%$ de $\mathrm{P}_{2} \mathrm{O}_{5}$ y $0.62 \%$ de $\mathrm{K}_{2} \mathrm{O}$. El día de la aplicación, el humus se diluyó con agua corriente hasta lograr conductividad eléctrica de $2.0 \mathrm{dS} \mathrm{m}^{-1}$. Se aplicó por aspersión un volumen promedio (en función de la densidad foliar) de $5 \mathrm{~L}$ por árbol hasta humedecer por completo las hojas. La aplicación se realizó con una bomba de aspersión manual en períodos de 14 días, para igualar la cantidad de nutrimientos aportados por el tratamiento químico.

A los árboles correspondientes, se aplicó, en dos periodos, la fórmula de fertilización de 750:250:250 para $\mathrm{N}, \mathrm{P}_{2} \mathrm{O}_{5}, \mathrm{~K}_{2} \mathrm{O}$, respectivamente. Los productos empleados fueron Triple-18® y fosfato de amonio, que fueron aplicados a cada árbol en surcos alrededor del tallo en la zona de goteo. Las aplicaciones se realizaron 14 días antes de iniciar y 14 días después de iniciada la etapa de florecimiento (LEITE et al., 2013).

Las variables de respuesta fueron evaluadas en diferentes etapas, a) inicio de florecimiento, b) inicio de fructificación, y c) cuando el fruto alcanzó la madurez comestible. Al iniciar la etapa de florecimiento, en 15 árboles por tratamiento (5 por bloque), se evaluó el número de inflorescencias por rama (considerando tres ramas por árbol) cada 14 días. Llegada la etapa de fructificación, en igual número de árboles, cada 14 días se cuantificó el número de frutos por inflorescencia.

Debido a que los arboles presentaban diferencias en la cantidad (volumen) de materia foliar, dada por la edad y las prácticas de poda, se procedió a realizar una estimación del volumen foliar individual (por árbol). Para tal determinación, se consideró la forma de cono truncado en la masa foliar de los árboles y mediante la fórmula $\mathrm{V}=\pi \cdot \mathrm{h}$ $\left(\mathrm{R}^{2}+\mathrm{r}^{2}+\mathrm{R} \cdot \mathrm{r}\right) 3^{-3}$, [donde $\mathrm{h}$ se refiere a la altura, $\mathrm{R}$ al radio inferior y $\mathrm{r}$ al radio superior], se obtuvo el volumen foliar $\left(\mathrm{m}^{3}\right)$. El volumen calculado se empleó como denominador para expresar los resultados de panículas, frutos y producción.

Al momento de cosecha, establecido por la desaparición del color verde de la piel del pseudofruto, se procedió a cortar la totalidad de los frutos (incluido el pseudofruto) y se registró la producción total. Tomando en cuenta la proporción que corresponde a cada parte del material colectado (fruto: pseudofruto) se estimó la proporción mostrada en la Tabla 2 y empleando este valor como factor se realizó la estimación del total de fruto producido. Para ambos casos los resultados se expresan como producción $(\mathrm{kg})$ por árbol y por $\mathrm{m}^{3}$ de volumen foliar. Adicionalmente se presenta la producción estimada por unidad de área cultivada $\left(\mathrm{kg} \mathrm{ha}^{-1}\right)$, considerando una densidad de siembra de 160 árboles por ha.

De manera paralela, de cada uno de los árboles previamente seleccionados para el muestreo, se tomaron aleatoriamente 10 frutos, y se trasladaron al laboratorio para su posterior análisis. Para evaluar las características físicas, se midió en fruto y pseudofruto: a) diámetro ecuatorial $(\mathrm{cm})$; b) longitud $(\mathrm{cm})$ con ayuda de vernier analógico y c) peso fresco (g) medido en balanza analítica.

Al jugo obtenido a partir del pseudofruto, se midió la acidez titulable (método 935.05 AOAC, 2010); contenido de sólidos solubles totales (SST) empleando refractómetro digital; azúcares reductores por el método DNS (AOAC, 2010); así como el contenido de polifenoles y la actividad antioxidante por el método de la decoloración del radical ácido 2,2'-azino-bis (3-etilbenzotiazolina-6-sulfónico) ABTS, ambos siguiendo los procedimientos descritos por De Lima et al. (2014).

Después de abrir mecánicamente los frutos, a las semillas se realizó las siguientes determinaciones: humedad (método 925.09), cenizas (método 923.03, grasa (método 920.39) mediante los métodos descritos por la AOAC (2010).

Los datos obtenidos se analizaron mediante el análisis de varianza y en donde existió diferencias se realizó la comparación de medias por el método de Duncan $(\alpha=0.05)$, empleando el programa infoStat $/ \mathrm{P}$, versión 2011.

\section{RESULTADOS}

Al inicio de la etapa de florecimiento (06 dic 2012) no se registró diferencia $(P>0.05)$ entre los tres tratamientos para la variable número de panículas por unidad de volumen foliar $\left(\mathrm{m}^{3}\right)$. A partir de ahí y durante todo el estudio, los tratamientos que fueron fertilizados, mostraron valores por encima del tratamiento sin fertilización (Figura 1). Durante los registros en las fechas 31 de enero y 14 de febrero, se observó incremento en la aparición de panículas para los tratamientos fertilizados, pero es más pronunciado donde se aplicó fertilización sintética. Incluso, el 14 de febrero se registró en promedio un valor de 3.64 panículas $\mathrm{m}^{-3}$ (el mayor valor de todo el estudio) para este tratamiento, siendo significativamente diferente a los otros dos tratamientos $(\mathrm{P}<0.05)$. Después del 
pico de floración observado esta fecha, se registró descenso paulatino para esta variable, congruente con el final del proceso de florecimiento. Para la mayoría de las fechas, los dos tratamientos fertilizados fueron estadísticamente iguales $(\mathrm{P}>0.05)$ y diferentes al tratamiento que no recibió fertilización.

De manera similar a lo que ocurrió con el número de panículas, la tendencia del número de flores por panícula presentó el comportamiento en forma de campana con respecto al tiempo (Figura 2). Los valores más altos correspondieron a las fechas del 31 de enero al 28 de febrero. Sin embargo, para esta variable aparentemente no se encontró un efecto claro de los tratamientos, toda vez que durante la mayoría de los registros no se encontró diferencia significativa entre tratamientos $(\mathrm{P}>0.05)$. Solamente para las fechas 3 de enero, 17 de enero, 31 de enero y las dos últimas del estudio (23 de mayo y 20 de junio) se encontró diferencia entre tratamientos, siendo al final de estudio menor el número de flores para el tratamiento que no fue fertilizado.

La dinámica del número de frutos por unidad de volumen de la copa del árbol durante todo el estudio se representa en la Figura 3. Se muestra la presencia de un pico de fructificación el 20 de diciembre para los tres tratamientos. Sin embargo, el valor más alto en la misma fecha, se reporta para el tratamiento con fertilización orgánica, que fue estadísticamente diferente $(\mathrm{P}<0.05)$ y casi cinco veces superior al tratamiento no fertilizado. Durante todo el estudio, el menor número de frutos se registró en los árboles que no fueron fertilizados, solo para la fecha 3 de enero, el número de fruto resultó igual estadísticamente a los tratamientos con los dos tipos de fertilización $(\mathrm{P}>0.05)$.

Los valores encontrados en el número de frutos, repercutieron de manera directa en la producción, como se muestra en la Tabla 1. Los tratamientos bajo fertilización fueron numéricamente superiores al no fertilizado, pero solo el tratamiento con biofertilización, fue diferente estadísticamente $(\mathrm{P}<0.05)$ en cuanto a la producción total $(\mathrm{kg}$ árbol $\left.{ }^{-1}\right)$ y por volumen foliar $\left(\mathrm{kg} \mathrm{m}^{-3}\right)$, al tratamiento que no fue fertilizado. Sobresale que cuando la comparación se realiza bajo condiciones más balanceadas (rendimiento por volumen foliar) el valor promedio obtenido de la producción del tratamiento biofertilizado $\left(0.30 \mathrm{~kg} \mathrm{~m}^{-3}\right)$ es tres veces superior al no fertilizado $\left(0.10 \mathrm{~kg} \mathrm{~m}^{-3}\right)$ para la producción total y de más del doble para la producción de frutos (calculada) por volumen foliar $\left(0.025 \mathrm{~kg} \mathrm{~m}^{-3}\right.$ en el biofertilizado) contra los dos tratamientos que fueron fertilizados. Además, estos mostraron para todas las variables un comportamiento similar $\left(0.07 \mathrm{~kg} \mathrm{~m}^{-3}\right.$ en el tratamiento sin fertilización). Para la cantidad de fruto por área cultivada estimada $\left(\mathrm{kg} \mathrm{ha}^{-1}\right)$ el tratamiento fertilización orgánica presentó valores superiores sobre los otros tratamientos, destacando el efecto positivo en la producción.

En la Tabla 2 se muestran las características físicas evaluadas a frutos y pseudofrutos. Solamente para la variable diámetro del pseudofruto se encontró diferencia significativa entre tratamientos $(\mathrm{P}<0.05)$, siendo el tratamiento con fertilización sintética diferente del no fertilizado, aunque este último tuvo un valor igual estadísticamente $(\mathrm{P}>$ 0.05) al tratamiento con fertilización orgánica. Se muestra también el valor de la proporción que ocupa el fruto respecto de todo el material colectado (fruto y manzana) y se puede ver que el tratamiento biofertilizado tiene numéricamente (no es posible aplicar comparación estadística, por tratarse de datos estimados) el factor con el valor mayor (0.082) seguido del tratamiento sin fertilización y finalmente del tratado con insumos sintéticos.

De igual forma, en la Tabla 3 se muestran las características bromatológicas y fisicoquímicas evaluadas a frutos frescos y pseudofrutos. De las variables evaluadas para fruto fresco, solamente el contenido de grasa presentó diferencia estadística $(\mathrm{P}<0.05)$ al presentar los tratamientos BIO y FS valores superiores al tratamiento sin fertilización. Para las evaluaciones realizadas al pseudofruto, el tratamiento biofertilizado presentó valores superiores y diferentes estadísticamente $(\mathrm{P}<0.05)$ al tratamiento no fertilizado para las variables contenido de acidez titulable y contenido de polifenoles totales (equivalentes de ácido gálico, EAG). Para la acidez titulable, los tratamientos fertilizados (sintética y biológica) mostraron igualdad estadística $(\mathrm{P}>0.05)$, sin embargo, la biofertilización logró aumentar en $81 \%$ el contenido de polifenoles respecto al tratamiento no fertilizado y casi $50 \%$ comparado con el fertilizado sintéticamente. Para las demás variables no se presentó diferencia estadística $(\mathrm{P}<0.05)$ entre los tres tratamientos.

\section{DISCUSIÓN}

Los resultados de este estudio muestran que la adición de nutrimentos en los tratamientos con algún tipo de fertilización, promovieron la aparición más temprana y mayor número de panículas en los árboles (Figura 1). Esto mismo ocurre en otras plantas, donde la fertilización orgánica aumentó el número de flores (SAIKIA et al., 2015). Sin embargo, no ocurrió lo mismo con el número de flores por panícula, donde todos los tratamientos presentaron valores similares 
(Figura 2). Se ha reportado que la producción de flores es sensible a varios factores precosecha. El riego de plantas adultas de marañón (OLIVEIRA et al., 2003) hace que se produzcan hasta $10 \%$ más flores por panícula. Lo anterior lleva a pensar que los árboles tratados en el presente estudio, pese a tener disponibilidad de nutrientes pudieron haber sufrido déficit hídrico. Este fenómeno pudo limitar la producción de flores (para todos los tratamientos), pero no ejerció efecto en la producción de frutos como se discute más adelante. De lo anterior, se demuestra que la fertilización no ejerce efecto sobre el número de flores por panícula.

Sin embargo, los valores encontrados en la cantidad de flores por panícula (valor máximo $\sim 100$ ) se encuentran por debajo del mínimo reportado que varía en el rango de 200 a 1600 flores por panícula (ERADASAPPA; MOHANA, 2016). Esto puede deberse a una característica particular de la variedad que se cultiva en la región (genotipo) o bien, que las condiciones del suelo y/o del clima no resultan las más apropiadas para promover mayor número de flores ya que se sabe que la respuesta de las plantas está muy ligada a las condiciones del suelo y/o el ambiente donde se establezcan. En nuestro estudio, los árboles están plantados muy cerca de la línea costera, lo cual pudo limitar la población y la actividad de los microrganismos benéficos asociados a la rizosfera. El efecto positivo que ejerce la fertilización orgánica en las plantas no solo se debe a que son una fuente orgánica de nutrientes, sino que además son capaces de promover y/o estimular el aumento de la población y de la actividad microbiana del suelo, como consecuencia de que regulan el proceso de solubilización de minerales y la mineralización de la materia orgánica (SUGINO et al., 2013).

Aunque siempre existe relación entre el número de flores por planta y el rendimiento poscosecha de las plantas, en los árboles de marañón tratados en este estudio no ocurrió así (Figuras 1, 2; Tabla 1). La variable más influenciada por la adición de nutrimentos (ambos tipos de fertilización) fue la producción de frutos; los valores obtenidos a lo largo del estudio fueron siempre superiores comparados con el tratamiento no fertilizado (Figura 3). Lo anterior demuestra que antes del estudio, los árboles se encontraban subnutridos, pues al adicionar formas disponibles de nitrógeno (en forma nítrica o amoniacal), fósforo intercambiable, potasio soluble, calcio y magnesio, se aumentó el número de frutos. Sin embargo, los fertilizantes orgánicos además de aportar nutrientes, mejoran las propiedades y estructura del suelo, promueven mayor disponibilidad de elementos minerales y el incremento de poblaciones microbianas y sustancias metabólicamente activas, como reguladores de crecimiento vegetal (BOWLES et al., 2014).

A pesar de que el tratamiento fertilizado orgánicamente presentó en algunas fechas menor proliferación de panículas, en comparación con el tratamiento de fertilización sintética, logró obtener mayor cantidad de frutos; dejando ver claramente el efecto ejercido sobre el fenómeno denominado "amarre" de los frutos, por lo que se hipotetiza que los biofertilizantes proporcionan nutrientes más disponibles a los árboles y mejoran su capacidad de transformar a tejidos vertedero. Ali et al. (2014) plantearon que la adición de fertilizantes orgánicos en cultivo de tomate (Solanum lycopersicum L.) aumentan la actividad biológica del suelo, facilitando el desarrollo radical de las plantas, el crecimiento del tallo, las hojas y mayor número de ramas, lo que atribuyen a la capacidad estimulante de los productos orgánicos así como de auxinas que ejercen efecto en el metabolismo, estimulan el crecimiento, desarrollo y rendimiento. Además los biofertilizantes han mostrado ser efectivos al disminuir la incidencia de patógenos y plagas del suelo que afectan el desarrollo óptimo de los cultivos y deterioran la calidad de los frutos. A pesar de que la planta con la que se compara es de arquitectura totalmente diferente, el fenómeno sobre el metabolismo y fisiología puede ser similar.

La producción de frutos fue numéricamente superior en los tratamientos con algún tipo de fertilización, pero solo el tratamiento con fertilización orgánica, fue diferente estadísticamente en cuanto al rendimiento por planta, por unidad de área foliar y por hectárea al tratamiento que no fue fertilizado (Tabla 1). De manera similar sucedió en cultivos de tomate (ton ha ${ }^{-1}$ ) donde la producción de frutos, además de mayor tamaño (longitud, diámetro y peso), fue favorecida al aplicar lixiviado de vermicompost de manera foliar en las primeras etapas de desarrollo y crecimiento de las plantas, comparado con tratamientos sin fertilización (ALI et al., 2014). Así mismo, se ha reportado que la aplicación de vermicompost puede incrementar el rendimiento y número de frutos de diversos cultivos. Similar a lo encontrado en este estudio, Ávila-Juárez et al. (2015), demostraron el efecto positivo de la aplicación de lixiviados de vermicompost, en la estructura del suelo, además del incremento en el rendimiento y la calidad nutricional de frutos de tomate.

Muchos trabajos se han enfocado a cultivos anuales, lo que pone de manifiesto que bajo condiciones de invernadero, la aplicación de diversos fertilizantes orgánicos promueve el crecimiento de 
cultivos anuales (YAN et al., 2015), además que estimula el aumento de biomasa radicular con el consecuente efecto sobre los productos obtenidos (JANNOURA et al., 2014). Aun cuando los resultados de producción de frutos y pseudofruto de marañón con fertilización orgánica son positivos, puede requerirse de más tiempo para obtener resultados claramente visibles, debido a que el crecimiento y desarrollo vegetativo es más lento (DENDENA; CORSI, 2014). Así se ha demostrado que ocurre en árboles de nectarina, donde se reporta que la aplicación reiterada de compost (por 3 años seguidos), aumentó la proliferación de raíz y la vida productiva de los árboles en comparación de suelos no tratados (BALDI et al., 2010).

A pesar de que en el presente estudio también se incluyó un tratamiento con fertilización sintética, este no repercutió en valores más altos en la producción respecto al tratamiento con fertilización orgánica, debido posiblemente a que el nitrógeno sintético favorece el desarrollo rápido de follaje pero no de tejidos vertedero. Al respecto, también se menciona que los fertilizantes orgánicos pueden estar proporcionando microelementos responsables de la movilización de macromoléculas desde las hojas hacia los frutos, de tal forma que favorecen una buena disponibilidad de este nutriente, lo que resulta decisivo para la obtención de alto rendimiento y calidad.

Respecto a las características morfológicas de los frutos, no se encontró diferencia significativa entre tratamientos en cinco de las seis variables evaluadas. La principal razón de esta ausencia de efecto es el genotipo, ya que se reporta que el tamaño de frutos está regulado genéticamente y cambios en esta característica son más bien el resultado de procesos de adaptación, domesticación o evolución, más que de la adición de compuestos durante la fertilización. La única característica morfológica que resultó diferente $(\mathrm{P}<0.05)$ fue el diámetro del pseudofruto (manzana), la cual seguramente posee mayor plasticidad fenotípica y está influenciada por el ambiente y las prácticas agronómicas empleadas.

La composición química de un fruto está relacionada de manera directa o indirecta con las características sensoriales o funcionales que habrá de exhibir, y esto a su vez es un indicio de pronta comercialización en función de las moléculas de interés que posea. En este sentido, las semillas de marañón provenientes de los arboles bajo fertilización presentaron contenidos superiores de grasa (Tabla 3 ). Esta característica le confiere a estas semillas mayor valor comercial, pues el contenido de grasa es una característica altamente deseable en la industria de producción de productos o derivados alimenticios. Se ha reportado que la semilla de marañón contiene $48 \%$ de grasa (RICO et al., 2015), sin embargo los valores de grasa encontrados en este estudio están muy por debajo de lo reportado (Tabla 3). Lo anterior puede deberse a múltiples factores, como la variedad del cultivo, las características del suelo, clima, tipo e intensidad de fertilización. Este último factor parece ser determinante, pues la plantación evaluada tiene un historial de no uso de fertilización (de ningún tipo) $\mathrm{y}$ al incorporarse nutrientes (de origen biológico o sintético) las plantas acumularon más lípidos en sus semillas. Una incorporación más prolongada de nutrientes al suelo y a la planta (fertilización por más de tres ciclos) podría verificar si el contenido de grasa puede igualar al obtenido en otras latitudes. Al parecer el uso de estos fertilizantes orgánicos promovieron las interacciones establecidas por las plantas con su medio biológico, químico y físico. Es presumible que en el ambiente rizosférico de los arboles fertilizados orgánicamente exista una mayor cantidad de biomoléculas que pueden ser absorbidas por la raíz, y posteriormente ser traslocadas a puntos específicos de la planta. Estas moléculas pueden ser incorporadas directamente en las vías biosintéticas del vegetal, por lo que este último ya no requiere gastar energía ni carbono en su síntesis. También es posible que alguna de estas biomoléculas sea activadora de los procesos de síntesis de lípidos.

También se encontró mayor contenido de acidez titulable y polifenoles (metabolitos secundarios) para los pseudofrutos de las plantas bajo fertilización orgánica; se piensa que la adición de nutrientes promovió la producción de metabolitos secundarios en las plantas, moléculas que pueden pertenecer a grupos de compuestos colorantes, aromáticos, reguladores del crecimiento y protectores naturales frente a parásitos, los cuales pueden tener un impacto significativo en el desarrollo vegetal (MARDANI-TALAEE et al., 2016). Sin embargo, aun cuando el contenido de compuestos de naturaleza fenólica fue superior, se encontró igual capacidad antioxidante para los pseudofrutos obtenidos de los tres tratamientos $(\mathrm{P}>0.05)$. Esto podría deberse a que los compuestos presentes en los pseudofrutos no fueron cuantificados o extraídos por el método por el que se evaluó la actividad antioxidante (la decoloración del ABTS), pero que si pueden contribuir con la actividad antioxidante total (si se evalúa ésta por varios métodos).

Desde la perspectiva nutricional, en un fruto es deseable un mayor contenido de compuestos de naturaleza fenólica, los cuales incluso pueden ser caracterizados como super-frutos por su 
importante aporte a la capacidad antioxidante y como atrapadores de radicales libres. Por el contrario, desde la perspectiva sensorial pueden resultar poco deseables en frutos dulces, pues imparten astringencia y amargor en los alimentos. Aun cuando en este estudio se encontró un valor de $394.37 \mathrm{mg}$
EAG $100 \mathrm{~mL}^{-1}$ para el tratamiento BIOF, superior respecto a la cantidad de polifenoles de los otros dos tratamientos, este valor está dentro del rango reportado para otras variedades en diversas regiones del mundo, donde se reportan valores de $338.6 \mathrm{mg}$ EAG $100 \mathrm{~mL}^{-1}$ (DE LIMA et al., 2014).

TABLA 1- Producción promedio obtenida y estimada para las tres estrategias de fertilización en plantas de marañón en etapa productiva durante un ciclo productivo.

\begin{tabular}{|c|c|c|c|c|c|c|}
\hline Producción & \multicolumn{2}{|c|}{$\begin{array}{c}\text { Fertilización } \\
\text { orgánica }\end{array}$} & \multicolumn{2}{|c|}{$\begin{array}{l}\text { Fertilización } \\
\text { sintética }\end{array}$} & \multicolumn{2}{|c|}{$\begin{array}{c}\text { Sin } \\
\text { fertilización }\end{array}$} \\
\hline \multicolumn{7}{|l|}{ Total (fruto y pseudofruto) } \\
\hline $\mathrm{kg}$ ábol $^{-1}$ & 21.95 & $\mathrm{~b}$ & 20.11 & $a b$ & 14.82 & $\mathrm{a}$ \\
\hline $\mathrm{g} \mathrm{m}^{-3}$ de materia foliar & 300 & $\mathrm{~b}$ & 270 & $\mathrm{ab}$ & 100 & $\mathrm{a}$ \\
\hline \multicolumn{7}{|l|}{ De fruto (calculada*) } \\
\hline $\mathrm{kg}$ árbol ${ }^{-1}$ & 1.80 & & 1.35 & & 1.09 & \\
\hline $\mathrm{g} \mathrm{m}^{-3}$ materia foliar & 25 & & 18 & & 7 & \\
\hline Total por área cultivada estimada $\left(\mathrm{kg} \mathrm{ha}^{-1}\right)$ & 3512 & & 3217.60 & & 2371.20 & \\
\hline Fruto por área cultivada estimada $\left(\mathrm{kg} \mathrm{ha}^{-1}\right)$ & 288 & & 216 & & 174.4 & \\
\hline
\end{tabular}

a-b Valores con letras diferentes en la misma fila denotan diferencia estadística significativa $(\mathrm{P}<0.05)$.

*el valor de la producción total fue multiplicado por el factor mostrado en la Tabla 2

TABLA 2 - Características físicas de frutos y pseudofrutos de marañón cosechados de tres estrategias de fertilización.

\begin{tabular}{lccc}
\hline \multicolumn{1}{c}{ Parámetro } & $\begin{array}{c}\text { Fertilización } \\
\text { orgánica }\end{array}$ & $\begin{array}{c}\text { Fertilización } \\
\text { sintética }\end{array}$ & $\begin{array}{c}\text { Sin } \\
\text { fertilización }\end{array}$ \\
\hline Peso del fruto $(\mathrm{g})$ & $10.27 \mathrm{a}$ & $9.60 \mathrm{a}$ & $10.71 \mathrm{a}$ \\
Ancho del fruto $(\mathrm{cm})$ & $2.10 \mathrm{a}$ & $2.23 \mathrm{a}$ & $2.10 \mathrm{a}$ \\
Largo del fruto $(\mathrm{cm})$ & $3.42 \mathrm{a}$ & $3.21 \mathrm{a}$ & $3.19 \mathrm{a}$ \\
Peso pseudofruto $(\mathrm{g})$ & $114.87 \mathrm{a}$ & $133.75 \mathrm{a}$ & $135.10 \mathrm{a}$ \\
Diámetro del pseudofruto $(\mathrm{cm})$ & $5.13 \mathrm{ab}$ & $5.55 \mathrm{~b}$ & $5.08 \mathrm{a}$ \\
Largo del pseudofruto $(\mathrm{cm})$ & $7.61 \mathrm{a}$ & $7.85 \mathrm{a}$ & $7.97 \mathrm{a}$ \\
Proporción peso de fruto/peso total & 0.082 & 0.067 & 0.073 \\
(fruto y pseudofruto) & & & \\
\hline
\end{tabular}

a-b Valores con letras diferentes en la misma fila denotan diferencia estadística significativa $(\mathrm{P}<0.05)$.

TABLA 3 - Características fisicoquímicas y bromatológicas de frutos y pseudofrutos de marañón cosechados de tres estrategias de fertilización.

\begin{tabular}{cccc}
\hline Parámetro & Fertilización orgánica & $\begin{array}{c}\text { Fertilización } \\
\text { sintética }\end{array}$ & $\begin{array}{c}\text { Sin } \\
\text { fertilización }\end{array}$ \\
\hline
\end{tabular}

\section{Fruto}

Humedad $\left(\mathrm{g} 100 \mathrm{~g}^{-1}\right)$

$21.09 \mathrm{a}$

$19.24 \mathrm{a}$

$18.95^{\mathrm{a}}$

Cenizas $\left(\mathrm{g} 100 \mathrm{~g}^{-1}\right)$

$3.05 \mathrm{a}$

$3.37 \mathrm{a}$

$3.25^{\mathrm{a}}$

Grasa $\left(\mathrm{g} 100 \mathrm{~g}^{-1}\right)$

$32.45 \mathrm{~b}$

$32.70 \mathrm{~b}$

$20.43^{\mathrm{a}}$

Pseudofruto (jugo)

Acidez titulable (g $\left.100 \mathrm{~g}^{-1}\right)$

$5.34 \mathrm{~b}$

$10.16 \mathrm{a}$

$4.84 \mathrm{ab}$

$4.06^{\mathrm{a}}$

SST $\left({ }^{\circ}\right.$ Brix $)$

$12.95 \mathrm{a}$

$10.51 \mathrm{a}$

$10.20^{\mathrm{a}}$

Cap. antioxidante $(\mathrm{mmol} \mathrm{ET} \mathrm{L-1})$

$184.59 \mathrm{a}$

$12.86 \mathrm{a}$

$12.54 \mathrm{a}$

Azúcares reductores $\left(\mathrm{mmol} \mathrm{L}^{-1}\right)$

$394.37 \mathrm{~b}$

$221.06 \mathrm{a}$

$214.68 \mathrm{a}$

Polifenoles totales (mg EAG $100 \mathrm{~mL}^{-1}$ )

$266.12 \mathrm{a}$

$217.84 \mathrm{a}$

a-b Valores con letras diferentes en la misma fila denotan diferencia estadística significativa $(\mathrm{P}<0.05)$. SST $=$ sólidos solubles totales; $\mathrm{ET}=$ equivalentes de Trolox; $\mathrm{EAG}=$ equivalentes de ácido gálico. 


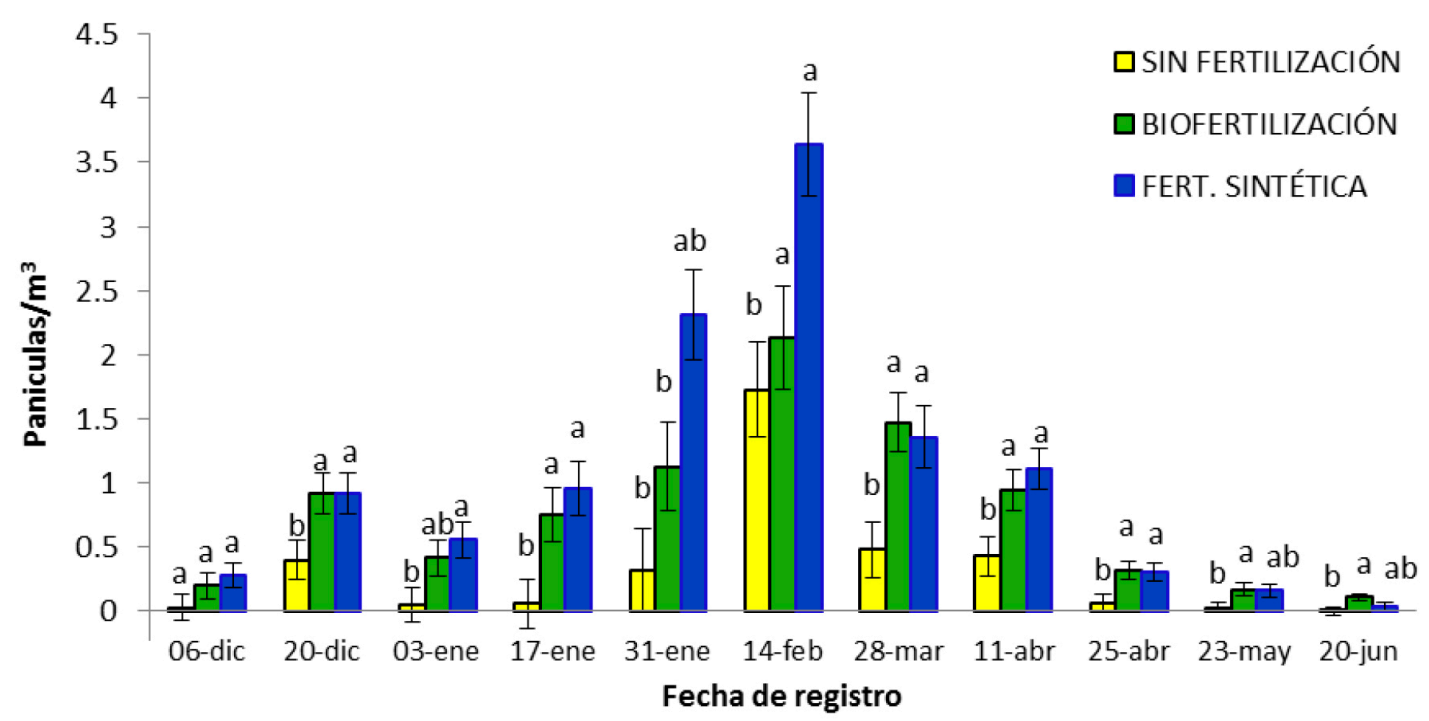

FIGURA 1- Panículas florales (pan $\mathrm{m}^{-3}$ ) en árboles de marañón (Anacardium occidentale L.) en etapa productiva (un ciclo) tratados con tres estrategias de fertilización. Cada valor es el promedio de: biofertilización $n=28$, fertilización sintética $n=28$, sin fertilización $n=32$. Las líneas sobre las barras muestran el error estándar.

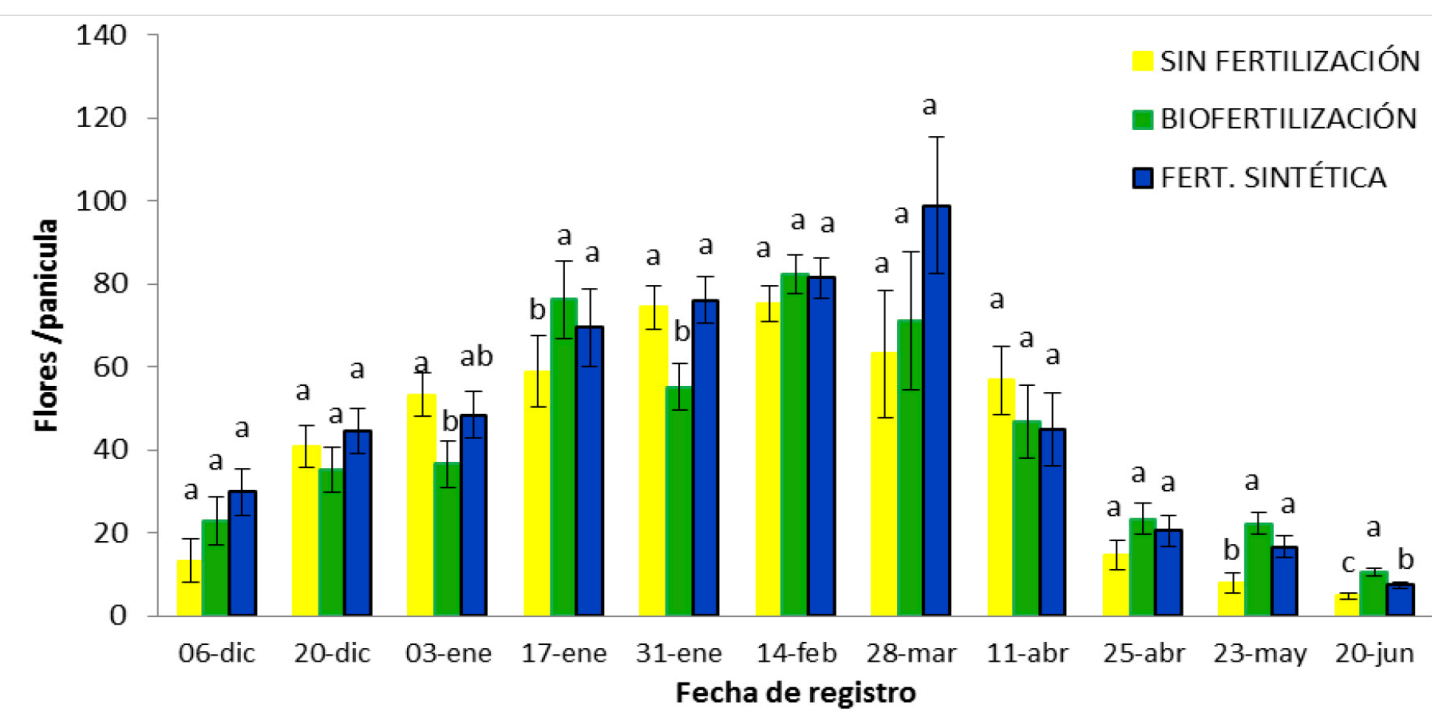

FIGURA 2- Número de flores por panícula en árboles de marañón (Anacardium occidentale L.) tratados con tres estrategias de fertilización durante el ciclo productivo de diciembre de 2012 a junio de 2033 Cada valor es el promedio de: biofertilización $n=28$, fertilización sintética $n=28$, $\sin$ fertilización $n=32$. Las líneas sobre las barras muestran el error estándar. 


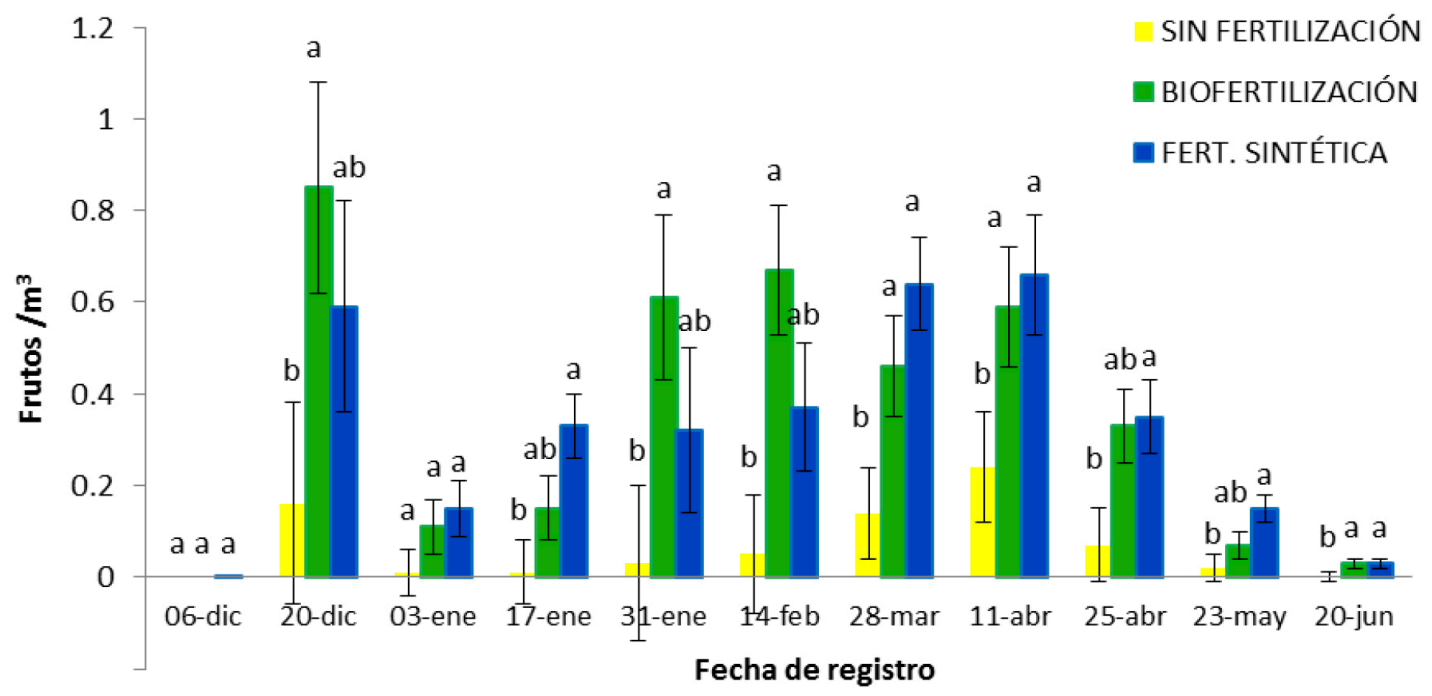

FIGURA 3- Dinámica de la producción de frutos de marañón en árboles tratados con tres estrategias de fertilización durante el ciclo productivo de diciembre 2012 a junio de 2013. Cada valor es el promedio de: biofertilización $n=28$, fertilización sintética $n=28$, sin fertilización $n=32$. Las líneas sobre las barras muestran el error estándar.

\section{CONCLUSIÓN}

En este trabajo se demostró que la aplicación de biofertilizantes por un ciclo productivo en plantas adultas de marañón (Anacardium occidentale L.) logró aumentar la producción de frutos en comparación con tratamientos de fertilización sintética y sin fertilización. De las características físicas evaluadas al pseudofruto (manzana), el diámetro de las manzanas biofertilizadas, resultó ser superior estadísticamente sobre los demás tratamientos. Así mismo, para frutos biofertilizados el análisis bromatológico reveló contenidos superiores de grasa y polifenoles totales, mejorando la calidad de los frutos cosechados.

\section{REFERENCES}

ADRIANO, M.L.; GUTIÉRREZ, F.; DENDOOVEN, L.; SALVADOR-FIGUEROA, M. Influence of compost and liquid bioferment on the chemical and biological characteristics of soil cultivated with banana (Musa spp. L.). Journal of Soil Science and Plant Nutrition, Temuco, v.12, n.1, p.33-43, 2012.
ALI, M.R.; MEHRAJ, H.; UDDIN, J. Foliar application of the leachate from vermicompost and mustard oil cake on the growth and yield of summer tomato. Middle-East Journal of Scientific Research, Dubai, v.22, n.8, p.1233-1237, 2014.

AOAC - Association of Official Analytical Chemists. HORWITZ, W. (Ed.). Official methods of analysis. $18^{\text {th }}$ ed. Gaithersburg, 2010.

ÁVILA-JUAREZ, L.; RODRÍGUEZ-GONZÁLEZ, A.; RODRÍGUEZ-PIÑA, N.; GUEVARAGONZÁLEZ, R.G.; TORRES-PACHECO, I.; OCAMPO-VELAZQUEZ R.V.; MOUSTAPHA, B. Vermicompost leachate as a supplement to increase tomato fruit quality. Journal of Soil Science and Plant Nutrition, Temuco, v.15 n.1, p.46-59, 2015.

BALDI, E.; TOSELLI, M.; MARCOLIN, G.; QUARTIERI, M.; CIRILLO, E.; INNOCENTI, A.; MARANGONI, B. Compost can successfully replace mineral fertilizers in the nutrient management of commercial peach orchard. Soil Use and Management, Hoboken, v.26, n.3, p.346-353, 2010. 
BOWLES, T.M.; ACOSTA-MARTÍNEZ, V.; CALDERÓN, F.; JACKSON, L.E. Soil enzyme activities, microbial communities, and carbon and nitrogen availability in organic agroecosystems across an intensively-managed agricultural landscape. Soil Biology and Biochemistry, Oxford, v. 68, p. 252262, 2014.

CATARINO, L.; MENEZES, Y., SARDINHA, R. Cashew cultivation in Guinea-Bissau - risks and challenges of the success of a cash crop. Scientia Agricola, Piracicaba, v.72 n.5, p. 459-467, 2015.

DE LIMA, A.C.S.; SOARES, D.J.; DA SILVA, L.M.R.; DE FIGUEIREDO, R.W.; DE SOUSA, P.H.M.; MENEZES, E.A. In vitro bioaccessibility of copper, iron, zinc and antioxidant compounds of whole cashew apple juice and cashew apple fibre (Anacardium occidentale L.) following simulated gastro-intestinal digestion. Food Chemistry, Oxford, v.161, p.142-147, 2014.

DEINLEIN, U.; STEPHAN, A.B.; HORIE, T.; LUO, W.; XU, G.; SCHROEDER, J.I. Plant salt-tolerance mechanisms. Trends in Plant Science, Londres, v.19, n.6, p.371-379, 2014

DENDENA, B.; CORSI, S. Cashew, from seed to market: a review. Agronomy for Sustainable Development, Paris, v.34, n.4, p.753-772, 2014.

ERADASAPPA, E.; MOHANA, G.S. Role of pollination in improving productivity of cashew $-\mathrm{A}$ review. Agricultural Reviews, Haryana, v.37. n.1, p, 61-65, 2016

GEISSELER, D.; SCOW, K.M. Long-term effects of mineral fertilizers on soil microorganisms - A review. Soil Biology and Biochemistry, Oxford, v.75, p.54-63, 2014

HERNÁNDEZ-FERNÁNDEZ, N.; ADRIANOANAYA, L.; SALVADOR-FIGUEROA, M.; BETANCUR-ANCONA, D.; VÁZQUEZ-OVANDO, A. Impact of organic fertilization on physicochemical and functional properties of cassava starch. Starch, Weinheim, v.68, n.5-6, p. 549-557, 2016.

JANNOURA, R.; JOERGENSEN, R.G.; BRUNS, C. Organic fertilizer effects on growth, crop yield, and soil microbial biomass indices in sole and intercropped peas and oats under organic farming conditions. European Journal of Agronomy, Amsterdam, v.52, p.259-270, 2014.
JAVANMARDI, J.; GHORBANI, E. Effects of chicken manure and vermicompost teas on herb yield, secondary metabolites and antioxidant activity of lemon basil (Ocimum $\times$ citriodorum Vis.) Advances in Horticultural Science, Firenze, v.26, n.3/4, p.151$157,2012$.

LEITE, K.N.; COSTA, R.N.T.; CRISÓTOMO, J.R.; FRIZZONE, J.A.; MARINHO, A.B. Economic analysis of cashew early dwarf Crop BRS - 189 depending on water levels and doses of potassium fertilization. Engenharia Agrícola, Jaboticabal, v.33, n.4, p.739-747, 2013.

MARDANI-TALAEE, M.; NOURI-GANBLANI, G.; RAZMJOU, J.; HASSANPOUR, M.; NASERI, B.; ASGHARZADEH, A. Effects of Chemical, organic and bio-fertilizers on some secondary metabolites in the leaves of bell pepper (Capsicum annuum) and their impact on life table parameters of Myzus persicae (Hemiptera: Aphididae). Journal of Economic Entomology, Cary, v.109 n.3 p.12311240,2016

OLIVEIRA, V.H.; BARROS, L.M.; LIMA, R.N. Influence of irrigation and genotype on the nut production of precocious dwarf cashew. Pesquisa Agropecuária Brasileira, Brasilia, DF, v.38 n.1, p.61-66, 2003.

RIBEIRO, L.R.; OLIVEIRA, L.M.; SILVA, S.O.; BORGES, A.L. Physical and chemical characterization of bananas produced in conventional and organic cultivation systems. Revista Brasileira de Fruticultura, Jaboticabal, v.34, n.3, p.774-782, 2012

RICO, R.; BULLÓ, M.; SALAS-SALVADÓ J. Nutritional composition of raw fresh cashew (Anacardium occidentale L.) kernels from different origin. Food Science and Nutrition, Hoboken, v.4, n.2, p.329-338, 2015.

SAIKIA, P.; BHATTACHARYA, S.S.; BARUAH, K.K. Organic substitution in fertilizer schedule: Impacts on soil health, photosynthetic efficiency, yield and assimilation in wheat grown in alluvial soil. Agriculture, Ecosystems and Environment, Amsterdam, v.203, p.102-109, 2015. 
SIAP - Servicio de Información Agroalimentaria y Pesquera. Produccion agrícola por cultivo, reporte 2014. Ciudad de México: SIAP, 2016. Disponível em: <http://www.siap.gob.mx>. Acesso em: feb. 2016.

SONG, X.H.; XIE, K.; ZHAO, H.B.; LI, Y.L.; DONG, C.X.; XU Y.C.; SHEN, Q.R. Effects of different organic fertilizers on tree growth, yield, fruit quality, and soil microorganisms in a pear orchard. European Journal of Horticultural Science, Leuven, v.77, n.5, p.204-210, 2012.

SUGINO, T.; NOBUNTOU, W.; SRISOMBUT, N.; RUJIKUN, P.; LUANMANEE, S.; PUNLAI, N. Effects of long-term organic material applications and green manure crop cultivation on soil organic carbon in rain fed area of Thailand. International Soil and Water Conservation Research, Beijing, v.1, n.3, p.29-36, 2013.
THIRKELL, T.J.; CAMERON, D.D.; HODGE, A. Resolving the 'nitrogen paradox' of arbuscular mycorrhizas: fertilization with organic matter brings considerable benefits for plant nutrition and growth. Plant, Cell and Environment, Hoboken, v.39, p.1683-1690, 2016.

XAVIER, F.A., MAIA, S.M.; ARAÚJO, R.K.; DE SÁ MENDOÇA, E.; DE OLIVEIRA, T.S. Effect of cover plants on soil $\mathrm{C}$ and $\mathrm{N}$ dynamics in different soil management systems in dwarf cashew culture. Agriculture, Ecosystems and Environment, Amsterdam, v.165, p.173-183, 2013.

YANG, B.; XIONG, Z.; WANG, J.; XU, X.; HUANG, Q.; SHEN, Q. Mitigating net global warming potential and greenhouse gas intensities by substituting chemical nitrogen fertilizers with organic fertilization strategies in rice-wheat annual rotation systems in China: A 3-year field experiment. Ecological Engineering, Amsterdam, v.81, p.289297, 2015. 